\title{
EL DEBATE DE ALFRED SCHÜTZ CON MAX SCHELER EN TORNO A LA EMPATÍA
}

\section{Alexis Emanuel Gros*}

Resumen: La empatía ha sido foco de discusión en los círculos antipositivistas de la academia alemana de comienzos del siglo pasado, especialmente dentro del movimiento fenomenológico. El presente trabajo se concentra en el debate en torno a esta problemática que Alfred Schütz sostiene con Max Scheler en Der sinnbafte Aufbau der sozialen Welt. En el primer apartado se bosquejan los lineamientos principales de la teoría scheleriana de la Fremdwabrnebmung (percepción del otro), y en el segundo, se exponen las críticas que Schütz le realiza a la misma sustentado en desarrollos teóricos de Edmund Husserl. La hipótesis que guía al escrito es que la confrontación con la teoría scheleriana de la Fremdwabrnebmung juega un rol fundamental en la configuración de la teoría del Fremdversteben (comprensión del otro) del Schütz temprano.

Palabras clave: empatía, fenomenología, intersubjetividad, Alfred Schütz, Max Scheler

Abstract: At the beginning of the $20^{\text {th }}$ century the issue of empathy was subject of controversy within the phenomenological movement. The present paper deals with the debate on that topic that the young Alfred Schutz maintained with Max Scheler. Firstly, I outline the Schelerian theory of Fremdwabrnebmung (perception of the other), and secondly I present Schutz's criticism of the former, which is inspired by Husserl's insights on self-awareness and Einfüblung (empathy). My thesis is that in order to properly understand Schutz's theory of Fremdverstehen (understanding of the other) it is essential to take into account this early confrontation with Scheler.

Keywords: Empathy, Phenomenology, Intersubjectivity, Alfred Schutz, Max Scheler

* Licenciado en Sociología (UBA) Becario doctoral del CONICET con sede de trabajo en el Instituto de Investigaciones Gino Germani (UBA) Ayudante de primera en la materia "Fenomenología Social" de la Carrera de Sociología (UBA). Dirección electrónica: alexisgros@hotmail.com 


\section{Introducción ${ }^{1}$}

La empatía ha sido foco de discusión y de interés en los círculos antipositivistas de la academia alemana de comienzos del siglo pasado, especialmente dentro del movimiento fenomenológico. Autores como Theodor Lipps, Wilhelm Dilthey, Edmund Husserl, Max Scheler, Aron Gurwitsch, Edith Stein y Alfred Schütz, entre otros, han dedicado sus esfuerzos a la investigación exhaustiva del problema, desarrollando teorías convergentes en ciertos aspectos y discordantes en otros.

En la actualidad podría hablarse de un renacimiento de la preocupación por esta problemática en la filosofía y las ciencias humanas. Es de destacar el trabajo que en los últimos años viene realizando el pensador danés Dan Zahavi, director del "Center for Subjectivity Research" de la Universidad de Copenhague, quien en escritos recientes ${ }^{2}$ se encarga de rehabilitar abordajes de representantes clásicos de la fenomenología, y de confrontarlos con desarrollos teóricos contemporáneos provenientes de la filosofía analítica y las neurociencias.

Siguiendo el impulso de Zahavi, me ocuparé en este escrito del debate en torno a la empatía que, en su primer obra fenomenológica, Der sinnhafte Aufbau der sozialen Welt, ${ }^{3}$ Alfred Schütz sostiene con Max Scheler. En general se relega la discusión Schütz-Scheler a un segundo plano en favor del

${ }^{1}$ Agradezco a la Dra. Graciela Ralón de Walton y al Dr. Carlos Belvedere por la atenta lectura de este escrito y por las sugerencias y comentarios que contribuyeron a darle su forma final. Debo mencionar también al Dr. Jochen Dreher y al Dr. Andreas Göttlich del Alfred-Schütz-Archiv de la Universität Konstanz, quienes durante mi estadía en Alemania me facilitaron material bibliográfico valioso en torno al debate Schütz-Scheler.

2 Cfr. Zahavi, Dan, "Empathy, Embodiment and Interpersonal Understanding: From Lipps to Schutz", en Inquiry, vol. 53, No 3, 2010.

${ }^{3} \mathrm{El}$ presente trabajo se concentra en las objeciones que Schütz le realiza a Scheler en su primer y único libro publicado en vida, Der sinnbafte Aufbau der sozialen Welt de 1932. No trataré aquí en profundidad las críticas del Schütz tardío a la teoría scheleriana de la Fremdwabrnebmung, las cuales se encuentran en un artículo de 1942 titulado "Scheler's theory of intersubjectivity and the general thesis of the alter ego". Cfr. Schutz, Alfred, "Scheler's theory of intersubjectivity and the general thesis of the alter ego", en A. Schutz, Collected Papers I. The problem of social reality, Martinus Nijhoff, The Hague, 1962. 
tratamiento del debate del sociólogo vienés con Max Weber. Esto se debe a que Schütz dedica casi la totalidad del mencionado libro a una crítica fenomenológica de la sociología comprensiva (verstehende Soziologie) weberiana, mientras que la figura de Scheler aparece sólo un puñado de veces y de forma marginal. En este trabajo intentaré rehabilitar esta disputa partiendo de la hipótesis de que la confrontación con la teoría scheleriana de la Fremdwabrnebmung (percepción del otro) ${ }^{4}$ juega un rol fundamental en la configuración de la teoría del Fremdversteben (comprensión del otro) del Schütz temprano.

Antes de comenzar con la exposición es preciso realizar una aclaración en referencia a la definición del concepto de empatía. Apoyado en Zahavi, me serviré aquí del término para señalar el acto de conciencia meramente cognitivo que nos brinda acceso a las vivencias ajenas. Este acto debe ser diferenciado tajantemente de la simpatía, que implica un compromiso afectivo del ego con el alter-ego, esto es, un compadecerse de la tristeza o congratularse de la alegría ajena. "La empatía y la simpatía, por cierto, no deben ser confundidas. Empatía (Empathie) (...) es el nombre para nuestra experiencia (Erfabrung) de las vivencias de otro. La simpatía supone más que sólo esto, aquí se trata, como ya lo señala la palabra, también de compasión (Mitgefübl)." 5

\section{Max Scheler: la teoría de la Fremdwabrnebmung}

A comienzos del siglo pasado dos teorías sobre la comprensión del alter-ego dominaban la escena académica alemana: la teoría del razonamiento por analogía (Analogieschlusstheorie) y la teoría de la proyección afectiva (Theorie der projektiven Einfühlung). En este contexto, cualquier pensador que quisiera esbozar una postura en torno a la empatía debía sentar posición frente a ellas. Es por ello que en la última parte de $W$ esen und Formen der Sympathie Max Scheler somete ambas teorías a una crítica fenomenológica para luego exponer su propio abordaje del problema.

${ }^{4}$ En lo concerniente a Max Scheler, me centraré exclusivamente en sus posiciones teóricas expresadas en Wesen und Formen der Sympathie, haciendo especial hincapié en las partes A - "Das Mitgefühl" -y C- "Vom fremden Ich".

${ }^{5}$ Zahavi, Dan, Phänomenologie für Einsteiger, W. Fink, Paderborn, 2007, p. 71. 
Estas teorías, en apariencia tan disímiles, tienen para Scheler un mismo punto de partida erróneo. Ambas se sustentan en dos presupuestos no revisados que se presentan como comprensibles de suyo y que, como tales, no resisten un análisis estrictamente fenomenológico. Estos supuestos son los siguientes: (I) en primer lugar, "que nos estaría 'dado' 'ante todo' sólo el yo propio" ("Es sei uns 'zunächst' immer nur das eigene Ich 'gegeben'") 6 y, (II) en segundo término, que "lo que nos estaría dado 'ante todo' de otro ser humano sería exclusivamente la aparición de su cuerpo, sus alteraciones, movimientos, etc." ("was uns von einem anderen Menschen 'zunächst' gegeben sei, das sei allein die Erscheinung seines Körpers, dessen Veränderungen, Bewegungen usw."). ${ }^{7}$ Podría afirmarse entonces que desde la perspectiva scheleriana la Analogieschlusstheorie y la Theorie der projektiven Einfüblung no son más que respuestas diferentes a una misma pregunta-problema que se desprende de los mencionados postulados: ¿cómo conocer los estados psíquicos del alterego si las únicas vivencias disponibles son las propias y lo único experimentable del otro es su cuerpo?

La Analogieschlusstheorie responde que para acceder a las vivencias del otro teniendo como única información los movimientos de su cuerpo es preciso llevar a cabo un razonamiento. Ego realiza movimientos corporales que de algún modo expresan sus vivencias. Puede inferir entonces que si alter-ego lleva a cabo un movimiento similar al suyo, estará experimentando una vivencia también análoga. Si por ejemplo ego ve a alter-ego "llorando" y quiere saber qué le sucede, lleva a cabo una deducción por analogía susceptible de ser expresada del siguiente modo: "cuando me siento triste, expreso este sentimiento a través del llanto; si el otro llora debe estar experimentando una tristeza similar a la que yo siento en esos casos".

Para Scheler esta teoría es unilateral porque hipostasia el modo, o uno de los modos, en que el hombre maduro y occidental conoce a sus congéneres, concibiéndolo como la única forma posible de empatía. Contra ella, el autor aduce que tanto los niños como los hombres de pueblos "primitivos" tienen una indudable capacidad de comprender al prójimo a

${ }^{6}$ Scheler, Max, Wesen und formen der Sympatbie, Francke, Bern, 1973, p. 238.

7 Ibid. 
pesar de carecer de la destreza necesaria para llevar a cabo operaciones intelectuales complejas. ${ }^{8}$

Por su parte, la Theorie der projektiven Einfüblung, elaborada por Theodor Lipps, es sumamente crítica de la Analogieschlusstheorie. 9 Lipps concibe a la empatía como un instinto, rechazando la idea de que sea necesaria una actividad intelectual para acceder a los estados psíquicos del otro. Cuando ego ve un gesto de alter-ego, tiene la tendencia a imitarlo; la imitación produce la aparición del estado psíquico asociado a dicho movimiento corporal, y esta vivencia evocada es finalmente "proyectada" o "transferida" al prójimo. Siguiendo los lineamientos de esta teoría, si veo a un hombre llorando debería ponerme a sollozar para comprender su tristeza, o si observo a un sujeto ahogándose en el mar, debería ahogarme yo mismo para entender su angustia y desesperación. Para Scheler, esto es absurdo. La experiencia nos enseña que podemos conocer los estados psíquicos ajenos sin necesidad de vivirlos en carne propia. Incluso podemos comprender vivencias de animales, ligadas a movimientos corporales que somos incapaces de imitar o reproducir. Así sucede por ejemplo cuando comprendemos la felicidad de un perro por el movimiento de su cola o su tristeza por sus aullidos. ${ }^{10}$

Una vez expuestas de forma sucinta las objeciones schelerianas a cada una de las teorías dominantes de la empatía, intentaré reconstruir la crítica al doble punto de partida (zweifacher Ausgangspunkt) común de las mismas. Esta crítica debe entenderse como una crítica fenomenológica. De acuerdo a Scheler, los presupuestos I y II deben ser descartados de cuajo porque son prejuicios tomados como comprensibles de suyo, y no conocimientos adquiridos en una investigación rigurosa limitada a describir lo dado en la intuición (Anschauung).

${ }^{8}$ Scheler esboza más argumentos en contra de esta teoría que no expondremos aquí. Para ahondar en estas críticas, Cfr. Ibid.

${ }^{9}$ Me centro aquí en la caracterización del pensamiento de Lipps que realiza Zahavi. Cfr. Zahavi, Dan, "Empathy, Embodiment and Interpersonal Understanding: From Lipps to Schutz", en Inquiry, vol. 53, No 3, 2010. Para la realización de la misma, Zahavi se sirve de un solo texto del autor: "Das Wissen von fremden Ichen" de 1907.

${ }^{10}$ Scheler, Max, op. cit. 
(I) El primer presupuesto afirma que "cada cual sólo puede pensar sus pensamientos [y] sentir sus sentimientos" ("dass jeder nur seine Gedanken denken, seine Gefüble füblen kann"),11 esto es, que cada sujeto sólo puede experimentar de forma directa sus propias vivencias, mientras que los estados psíquicos ajenos son de difícil o imposible acceso. Para Scheler este supuesto es erróneo en tanto se sustenta en una idea metafísica: que cada individuo tiene una conciencia monádica cerrada en sí misma, la cual no puede interpenetrarse o mezclarse con otras. Oponiéndose a esta idea, el autor señala que la experiencia nos enseña que "podemos pensar tanto 'nuestros' 'pensamientos' como los 'pensamientos' de otros, sentir (...) nuestros sentimientos tanto como los de otros."12 En efecto, nunca podemos estar completamente seguros de que nuestros pensamientos y sentimientos sean efectivamente nuestros. Muchas veces nos atribuimos como propias ideas que leímos en un libro, que escuchamos en boca de un amigo o que nos fueron inculcadas por nuestros educadores. Para Scheler, fenoménicamente no se nos donan conciencias monádicas sino una "conciencia omincomprensiva" (allumfassendes Bewusstsein), ${ }^{13}$ una corriente de vivencias suprapersonal de la que todos participamos y dentro de la cual es imposible distinguir qué vivencia pertenece a cada quien. “'Ante todo' se da curso una corriente de vivencias indiferente con respecto al yo y al tú (in Hinsicht auf Ich-Du indifferenter Strom der Erlebnisse), que encierra de hecho indistinto y mezclado lo propio y lo ajeno."14

Vivir inmerso en el alma colectiva es para Scheler una constante antropológica. “'Ante todo' vive el hombre más en los otros que en sí mismo; más en la comunidad que en su individualidad." 15 El fenomenólogo de Múnich sustenta nuevamente estas afirmaciones en desarrollos teóricos sobre la psiquis infantil y la vida "primitiva" de los pueblos. El niño no tiene individualidad ni autoconciencia, vive inmerso en el espíritu de su familia (familiärer Geist), se pierde extáticamente en su mundo psíquico circundante (psychische Umwelt) mamando como la leche materna las vivencias de sus padres y allegados. Sólo en una fase tardía de su maduración puede tomar

\footnotetext{
11 Ibid. p. 239.

12 Ibid. p. 238.

${ }^{13}$ Ibid. p. 244.

14 Ibid. p. 240.

${ }^{15}$ Ibid. p. 241.
} 
distancia de esta totalidad primigenia y acceder a una vida de conciencia propia, la cual sin embargo nunca puede desligarse completamente de la corriente psíquica universal. Algo similar sucede con el hombre "primitivo". Para Scheler, en un argumento que recuerda al Émile Durkheim de De la division $d u$ travail social, 16 el "primitivo" vive sumergido en la conciencia colectiva de su pueblo; su vida psíquica individual está fundida (eingeschmolzen) en el alma de la comunidad. Es por ello que ante un agravio a un miembro de su grupo o ante la violación de una norma comunitaria, reacciona violenta y vengativamente como si se tratara de un ataque hacia sí mismo. Se vislumbra así que de acuerdo a Scheler el surgimiento de la individualidad es un fenómeno tardío tanto en el desarrollo ontogenético como en el histórico. El individuo como mónada es producto de la modernidad europea. En sociedades "primitivas" el alejamiento de la vida común y el forjamiento de una personalidad individual y única son vistos como características patológicas, mientras que en el mundo moderno occidental es justamente la ausencia de estos rasgos subjetivos lo que se considera anormal.

Para finalizar con las objeciones de Scheler a este primer presupuesto es preciso dar cuenta de su tratamiento de la noción de percepción interna (innere Wabrnebmung). De acuerdo a desarrollos teóricos dominantes en la época -entre cuyos representantes se cuentan Wilhelm Dilthey y Edmund Husserl-, la percepción interna es un proceso de conciencia que nos brinda acceso a nuestros estados psíquicos de modo análogo a como la percepción sensible nos permite conocer objetos trascendentes. Según estas perspectivas, la percepción interna es eo ipso autopercepción, es decir, cada sujeto sólo tiene acceso a su propio flujo vivencial, siéndole inaccesible la corriente de conciencia ajena. Apoyado en la recién expuesta noción de conciencia omnicomprensiva, Scheler se opone a esta idea y señala que, en tanto partícipe de la vida psíquica universal, todo sujeto tiene acceso, al menos potencialmente, no sólo a sus propias vivencias sino también a las de sus prójimos.

${ }^{16}$ Cfr. Durkheim, Émile, La división del trabajo social, Ed. Libertador, Buenos Aires, 2004. 
En el sentido de nuestra interpretación tendríamos que decir: el acto de intuición interna (innere Anschaunng) de A [un individuo A] abarca no sólo los procesos de su propia alma (eigene Seelenvorgänge), sino en derecho y como posibilidad el reino entero existente de las almas (das gesamte existierende Reich der Seelen) - por lo pronto como una corriente todavía indistinta de vivencias (ungegliederter Strom von Erlebnisse). ${ }^{17}$

(II) El segundo presupuesto de las teorías de la empatía reza que lo único que puede experimentarse del alter-ego es su cuerpo. Para reconstruir la crítica scheleriana a este supuesto es necesario problematizar la recién introducida noción de percepción interna. Podría señalarse que el autor utiliza este último concepto sólo para discutir dentro del terreno de sus adversarios teóricos. En realidad, desde la perspectiva de Scheler, una percepción interna "pura", un acto que capte vivencias, ya sean propias o ajenas, descontaminadas de todo tipo de mediación corporal es una quimera. Incluso en lo que respecta a la autopercepción, resulta inviable concebir a la reflexión como lo hace Husserl, esto es, como un acto intencional que permite intuir (anschauen) inmediatamente los propios estados psíquicos. "Semejante autopercepción (Selbstwahrnebmung) puramente intra-anímica" (innerseelische) — dice Scheler - es una mera ficción."18 Cuando me percibo a mí mismo jamás se me dona algo así como un objeto inmanente "puro" de carácter meramente espiritual; lo que experimento es siempre una unidad expresiva (Ausdruckseinheit), una totalidad en la cual la vivencia y su correspondiente modo de expresión corporal están fundidos. Para decirlo en términos de Merleau-Ponty, la conciencia es en Scheler "inseparable de su expresión (...) no hay conciencia detrás de las manifestaciones, éstas son inherentes a la conciencia, ellas son la conciencia."19 Mi ira, por ejemplo, no es un objeto psíquico puro sino una totalidad expresiva que engloba mis gritos, mi sentimiento de irritación, el enrojecimiento de mi cara, mis movimientos atolondrados, etc. Si quiero conocer mi propio carácter, por tanto, sólo podré hacerlo contemplándome a mí mismo en acción,

17 Scheler, Max, op. cit., pp. 243-244.

${ }^{18}$ Ibid. p. 245.

${ }_{19}$ Merleau-Ponty, Maurice, Merleau-Ponty à la Sorbonne. Résumé de cours 1949-1952, Cynara, Paris, 1988, p. 43. 
expresándome, y no hundiéndome en una reflexión introspectiva como la husserliana.

A través de la introducción de la noción de unidad expresiva, Scheler puede enfrentarse con la convicción dominante de que existe una diferencia abismal entre la autopercepción y la comprensión del otro. Desde la óptica de Scheler tal hiato es inexistente porque ambos procesos funcionan de modo análogo: de mí mismo y del prójimo experimento sólo fenómenos expresivos. "En el fondo — dice Scheler- no existe, pues, en este punto absolutamente ninguna distinción tan radical entre la autopercepción y la percepción del otro (Selbst- und Fremdwabrnebmung)."20 Así como no es cierto que en la autopercepción se intuyan sólo vivencias puras, tampoco es cierto que en la experiencia del alter-ego se perciba un mero cuerpo. Un cuerpo de estas características sólo es experimentado por el forense cuando escudriña un cadáver o por el médico cuando analiza la pierna o el brazo de su paciente. 'La idea de que 'ante todo nos es dado sólo un cuerpo' (Körper) es completamente errónea. Sólo al médico o al investigador de la naturaleza se les dona algo así, esto es, al hombre que se abstrae artificialmente del fenómeno de expresión (Ausdrucksphänomen) que se dona en modo primario." 21 Es decir, tanto la idea de una vivencia pura como la de un cuerpo meramente material privado de significado espiritual son para Scheler abstracciones respecto del fenómeno originario de la expresión.

Siguiendo estos lineamientos, Scheler plantea su propia teoría de la comprensión del alter-ego: la teoría de la percepción del otro (Fremdwahrnehmung). Para conocer al prójimo, señala, no es preciso realizar ningún tipo de razonamiento ni llevar a cabo una proyección afectiva como la planteada por Lipps. "Debe ser resaltado que esta aprehensión y comprensión [la aprehensión y comprensión del prójimo] no se produce ni por medio de un razonamiento - 'razonamiento por analogía' (Analogieschluss) - ni a través de proyección afectiva (projektive 'Einfüblung) e impulsos de imitación."22 La experiencia del otro no es indirecta y compleja sino más bien simple e inmediata. Se trata de un acto cognitivo análogo a la percepción sensible, esto es, de un acto que nos brinda los objetos de un golpe

${ }^{20}$ Scheler, Max, op. cit., p. 245.

${ }^{21}$ Ibid. p. 21.

22 Ibid. p. 20. 
y en carne $y$ bueso. Las vivencias del alter-ego son percibidas por el ego inmediatamente; pero no como objetos psíquicos puros sino amalgamadas con movimientos corporales, formando parte de unidades expresivas. "El hecho de que existen 'vivencias' (Erlebnisse) nos es dado en los fenómenos de expresión (Ausdrucksphänomene) -una vez más, no a través de un razonamiento, sino 'inmediatamente'- en el sentido de una "percepción" originaria (originäres Wabrmebmen)." 23 Así, por ejemplo, jamás observo los ojos del prójimo en tanto mero fenómeno material; veo en ellos que el otro "me está mirando", que "me mira con descofianza", que "trata de esquivar mi mirada", etc. Así puede afirmarse que en la risa del alter-ego se percibe inmediatamente su alegría; en su llanto, su tristeza; en su rubor, su vergüenza, en el rechinar de sus dientes, su ira. En un pasaje ya clásico de Wesen und Formen der Sympathie Scheler resume su posición señalando: "Es seguro, en efecto, que creemos tener directamente en la risa la alegría, en el llanto la pena y el dolor del otro, en su rubor su vergüenza, en sus manos suplicantes, su suplicar, en la tierna mirada de sus ojos su amor, en su rechinar de dientes su furia, en su puño amenazante su amenazar, en el sonido de sus palabras la significación que menta, etc."

\section{Alfred Schütz: la teoría del Fremdversteben}

Al igual que Scheler, Alfred Schütz elabora su propia concepción de la empatía dialogando con teorías contemporáneas. Si bien es innegable que en Der sinnhafte Aufbau der sozialen Welt sus interlocutores principales son Max Weber y Edmund Husserl, creo que la poco tratada confrontación de Schütz con otros dos autores de habla alemana, Rudolf Carnap y Max Scheler, juega un rol decisivo en la configuración de su teoría del Fremdversteben. Schütz considera a Carnap y a Scheler representantes de dos posiciones extremas que deben ser evitadas si se pretende alcanzar una visión verdaderamente fenomenológica de la empatía. La postura schütziana en torno a la comprensión del otro puede ser entendida entonces como un intento de mantener el equilibrio entre dos errores simétricos: la afirmación de la inviabilidad de la empatía (Carnap) y la aseveración de la posibilidad de la empatía total (Scheler). 
Por un lado, Carnap sostiene que la empatía es imposible. El autor del Wiener Kreis coarta el campo de la experiencia reduciéndolo a la mera sensorialidad: lo único asequible del alter-ego son sus movimientos corporales en tanto objetos del mundo exterior, mientras que sus estados psíquicos permanecen siempre en la oscuridad dada su inaccesibilidad a los sentidos. Desde esta posición no sólo sería inviable la comprensión del otro en la vida cotidiana sino también la fundación de una disciplina como la sociología comprensiva weberiana, la cual no sería más que mera especulación metafísica, ya que resulta inviable generar saber científico a partir de hechos no experimentables. "Esta tesis lleva al siguiente resultado: la psiquis ajena no nos estaría dada de ningún modo, sino sólo cosas del mundo exterior (Dinge der Außenwelt), la suposición de una psiquis ajena sería solamente un elemento prescindible y secundario frente a lo físico, y las tesis sobre ella no tendrían ningún sentido científico..."24 Apoyado en Husserl, Schütz se enfrenta en Der sinnhafte Aufbau der sozialen Welt y en escritos posteriores a este cercenamiento del campo experiencial propiciado por el positivismo lógico. Para el fundador de la sociología fenomenológica es un hecho innegable que en la cotidianeidad comprendemos y experimentamos las vivencias del prójimo. Incluso los representantes del positivismo lógico, que con tanto ímpetu niegan la posibilidad de la empatía, deben apoyarse en ella una y otra vez en su trajín diario. Esto sucede por ejemplo cuando concurren a congresos especializados y se vinculan con otros filósofos y científicos, comprendiéndolos y siendo comprendidos por ellos. ${ }^{25}$

Por otro lado, como se desprende de lo desarrollado en el primer apartado, Scheler sostiene una posición diametralmente opuesta a la carnapiana. Para el fenomenólogo de Múnich, la empatía no sólo es posible sino que puede ser total (totale Einfüblung). En tanto partícipe de la conciencia omnicomprensiva, todo sujeto tiene acceso directo no sólo a sus vivencias sino también a las del prójimo. Como veremos más adelante, Schütz critica la idea scheleriana de una empatía total apoyado en las investigaciones husserlianas sobre las estructuras esenciales de la conciencia. "Ahora bien, la

${ }^{24}$ Schütz, Alfred, Der sinnbafte Aufbau der sozialen Welt, Suhrkamp, Frankfurt am Main, 1981, p. 29.

${ }^{25}$ Cfr. Schutz, Alfred, "Concept and theory formation in the social sciences", en A. Schutz, Collected Papers I. The problem of social reality, Martinus Nijhoff, The Hague, 1962. 
suposición de una empatía total (totale Einfüblung) es una hipótesis que, como se mostrará, está en conflicto con las leyes esenciales de la vida de la conciencia (Wesengesetzlichkeiten des Bewusstseinslebens)."26

Más arriba esbocé las objeciones de Scheler a los presupuestos comunes de las teorías dominantes de la comprensión del otro (I y II). En abierto enfrentamiento con Scheler, Schütz realiza en Der sinnhafte Aufbau der sozialen Welt una defensa fenomenológica de esos dos mismos postulados sustentada en desarrollos teóricos husserlianos. A continuación trataré de reconstruir dicha defensa poniendo especial énfasis en las discrepancias y tensiones entre las perspectivas de ambos pensadores.

(I) En Der sinnbafte Aufbau der sozialen Welt, Schütz reconoce explícitamente que Scheler está en lo cierto cuando afirma que "la realidad del 'tú' y de una comunidad está absolutamente predada (vorgegeben) al ser real del 'yo' en el sentido del yo-propio (Eigen-Icb) y lo 'auto-vivenciado' (Selbst-erlebten) singular e individualmente." 27 En efecto, como lo señala en un artículo de los años cuarenta dedicado a la teoría de la intersubjetividad de Scheler, Schütz coincide en cierta medida con el postulado scheleriano de que el "nosotros" precede al "yo": los seres humanos no vienen al mundo $e x$ nibilo sino que son paridos por madres y criados en el seno de grupos sociales. ${ }^{28}$ Sin embargo, para Schütz el "nosotros" no debe concebirse como una estructura objetiva que subsiste independientemente de la subjetividad individual. La esfera del "nosotros" remite siempre en última instancia al "yo", puesto que no es más que el correlato de los actos intencionales de un sujeto. Schütz se aferra aquí a uno de los rasgos básicos de la fenomenología husserliana: el respeto de la perspectiva de primera persona (erste Person Perspektive). ${ }^{29}$ Resulta inconducente hablar del "nosotros" como de una totalidad objetiva anterior al "yo" porque el "yo" debe estar de alguna manera presente para que el "nosotros" se manifieste como tal. Así como la izquierda y la derecha no son conceptos absolutos sino nociones relativas a

26 Schütz, Alfred, Der sinnbafte Aufbau der sozialen Welt, Suhrkamp, Frankfurt am Main, 1981, p. 29.

27 Ibid., p.137.

${ }^{28} \mathrm{Cfr}$. Schutz, Alfred, "Scheler's theory of intersubjectivity and the general thesis of the alter ego", en A. Schutz, Collected Papers I. The problem of social reality, Martinus Nijhoff, The Hague, 1962.

${ }^{29}$ Cfr. Zahavi, Dan, Pbänomenologie fïr Einsteiger, W. Fink, Paderborn, 2007. 
mi ubicación espacial, así también los pronombres personales "nosotros" y "ellos" remiten en última instancia a mi localización social. Se vislumbra así que a pesar de concebir al hombre como un ser eminentemente social, Schütz considera fundamental hacerle justicia a lo único e irrepetible de la experiencia individual. Es por ello que, sustentado sobre todo en Husserl, concibe a la intersubjetividad como inter-subjetividad, esto es, no como una totalidad hispostasiada a la manera de la Volksseele (alma del pueblo) de Herder, sino como un vínculo entre-sujetos. ${ }^{30}$ Los individuos pueden enlazarse entre sí con altos grados de intimidad, como sucede por ejemplo en la relación nosotros pura (reine Wirbeziehung), pero permanecen siempre individuos. Sus respectivas corrientes de conciencia jamás pueden fusionarse completamente. Por tanto, desde la posición de Schütz, lejos de verificarse en la experiencia, la unificación afectiva que según Scheler existe entre la madre y el niño, el "primitivo" y su tribu y la masa y el líder no es más que una idea metafísica.

Partiendo de este enfoque egológico de tinte husserliano, que por cierto resulta absolutamente compatible con el individualismo metodológico de Weber, Schütz se ocupa de resaltar el hiato existente entre el curso de conciencia del ego y el del alter-ego. Siguiendo a Husserl, el sociólogo vienés señala que cada sujeto es portador de una corriente de conciencia cerrada en sí misma que no puede mezclarse o interprenetrarse con otras. Cada individuo experimenta de forma directa y originaria el flujo vivencial propio, siendo imposible una experiencia de estas características de la conciencia ajena. Si, como parece indicar Scheler con su noción de conciencia omnicomprensiva, ego pudiese experimentar las vivencias de alter-ego de la misma manera en que éste lo hace, no habría entonces ninguna diferencia entre uno y otro. Tanto para Schütz como para Husserl, la brecha entre la conciencia propia y la ajena es un fenómeno pregnante que debe ser atendido y respetado. Así lo señala Husserl en un pasaje de las Cartesianische Meditationen: "Si ocurriera esto, si lo esencialmente propio del otro fuera accesible de modo directo, sería entonces mera parte no independiente de lo propio de mí mismo, y, en fin, él mismo y yo mismo seríamos uno". ${ }^{31}$

${ }^{30} \mathrm{La}$ idea de concebir a la intersubjetividad como inter-subjetividad pertenece a Zahavi. Cfr. Zahavi, Dan, Husserls Phänomenologie, Mohr Siebeck, Tübingen, 2009. ${ }^{31}$ Husserl, Edmund, Meditaciones cartesianas, Fondo de Cultura, México, 2004, p. 156. 
De acuerdo a Husserl, cada sujeto se experimenta a sí mismo de forma privilegiada en las dos modalidades fundamentales de la autoexperiencia: la autopercatación pre-reflexiva (pre-reflextive self-awareness) y la autoconciencia reflexiva. ${ }^{32}$ La primera de estas modalidades puede entenderse como un minimum de autoconciencia que actúa como presupuesto básico de toda subjetividad: sólo es sujeto quien se percata de sí mismo, quien "vive" sus propias vivencias. Este "experimentar" los propios estados psíquicos no implica sin embargo tematizarlos, esto es, dirigir la mirada reflexiva hacia ellos y constituirlos como objetos inmanentes; se trata más bien de un modo de experiencia de sí mismo en el que no existe distinción alguna entre sujeto aprehensor y objeto aprehendido, y que funciona pasivamente en toda actividad de la conciencia. En un texto reciente Zahavi señala que la autopercatación pre-reflexiva se identifica con la conciencia interna del tiempo (inneres Zeitbewusstsein) y su fase triádica retención, impresión originaria y protención. Siguiendo esta sugerencia del pensador danés, podría señalarse que aquello de lo que uno se percata pasivamente no es el presente puntual sino un presente especioso (specious present $)^{33}$ en el que se retienen las vivencias recién pasadas y se anticipan las vivencias por venir. De acuerdo a Husserl, esta modalidad de la autoexperiencia es la que se posee mientras transcurren los actos conscientes. Cuando están en proceso, las vivencias intencionales son pre-fenoménicas, puesto que el rayo de la mirada no se dirige hacia ellas sino al objeto intencionado; el acto, por el contrario, no "se ve" sino que meramente "se vive". Sólo a posteriori, luego de que el estado psíquico en cuestión ha transcurrido, adviene el segundo modo de autoexperiencia: la autoconciencia reflexiva, en la cual la conciencia se escinde a sí misma y realiza un giro de la mirada (Blickwendung) de la trascendencia a la inmanencia, iluminando y apresando a las vivencias recién pasadas. Podría afirmarse con Zahavi que "Husserl (...) tomaba a la autopercatación (self-awareness) como una característica esencial de la subjetividad y consideraba a la reflexión como una forma fundada y no básica de autoconciencia". ${ }^{34} \mathrm{La}$ autoconciencia

${ }^{32}$ Zahavi, Dan, "Inner time-consciousness and pre-reflective self-awareness", en D. Welton (ed.), The new Husserl. A Critical Reader, Indiana University Press, Indiana, 2003.

${ }^{33}$ El concepto de specious present pertenece a William James. Cfr. James, William, The Principles of Psychology. Holt and Company, New York, 1890.

${ }^{34}$ Ibid., 158. 
reflexiva está fundada en la autopercatación pre-reflexiva porque para que la reflexión tenga algo hacia lo que dirigirse y no sea un mero discurrir en el vacío, las vivencias recién pasadas deben permanecer almacenadas de algún modo en la conciencia. Esto es posibilitado por la retención: los estados psíquicos pasados se conservan en el presente especioso pero sometidos a la modificación retencional, la cual los hunde progresivamente en la opacidad. Es gracias a este proceso que pueden ser recuperados reflexivamente en cualquier momento ulterior de la vida consciente.

Veamos las consecuencias que tienen estas consideraciones husserlianas para nuestro problema: yo "vivo" mis estados psíquicos y los retengo en mi flujo de conciencia; y dado que los tengo siempre a disposición en tanto retenidos, puedo en cualquier momento fijar mi atención sobre ellos y reactivarlos a través de actos de percepción interna. Por el contrario, el alter-ego no tiene acceso a mi conciencia en ninguna de estas dos modalidades. No puede "vivir" mis vivencias; dado que no las "vive", no puede retenerlas, y, en consecuencia, no está capacitado para reflexionar sobre las mismas.

En Der sinnhafte Aufbau der sozialen Welt, Schütz se sirve de los mencionados desarrollos husserlianos para reformular la teoría de la acción de Max Weber. Weber señala en Wirtschaft und Gesellschaft que la acción (Handeln) es un comportamiento al cual el actor le enlaza un sentido subjetivo (subjektiver Sinn). "Llamamos ‘acción (Handeln) al comportamiento humano $(. .$.$) en la medida en que el agente o los agentes vinculen a aquel$ comportamiento un sentido subjetivo". 35 Sin embargo, no analiza en profundidad el proceso de dotación de sentido llevado a cabo por la conciencia subjetiva. A Schütz le interesa esclarecer este proceso, que denomina autointerpretación (Selbstauslegung), dando cuenta de las estructuras de la conciencia que actúan en él. Articulando desarrollos husserlianos con elementos de la Lebensphilosopbie de Henri Bergson, Schütz señala de forma similar a Husserl que existen dos modos básicos de experiencia de sí, cada uno de los cuales se corresponde con uno de los extremos de la clásica antinomia vitalista: "vida-pensamiento" (LebenDenken). La primera modalidad de autoexperiencia, correspondiente al extremo "vida", es la que tiene lugar durante el transcurso del 
comportamiento y se identifica para Schütz con la durée bergsoniana. En ella el yo es uno con su flujo vivencial; se entrega o se deja llevar por el mismo. Sus vivencias particulares se le manifiestan no como claras y distintas sino que "se funden unas con otras en una transición continua." 36 Cuando se vive en la durée, dice Schütz, "no hay Nebeneinander (estar-uno-junto-a-otro), Außereinander (estar-uno-afuera-del-otro) ni divisibilidad (Teilbarkeit) [de las vivencias], sino sólo la continuidad del fluir." 37 Puede verse claramente que esta forma de autoexperiencia es homologable con la recién mencionada autopercatación pre-reflexiva husserliana. Ambas se desarrollan mientras el acto intencional está teniendo lugar y en ambas no existe una diferenciación neta entre sujeto aprehensor y objeto aprehendido. Es decir, tanto en la autopercatación husserliana como en el "dejarse vivir en la durée" schütziano los estados psíquicos meramente "se viven" pero no se captan de forma atenta.

Schütz considera que el proceso de dotación de sentido de la propia conducta sólo puede tener lugar en el marco de una segunda modalidad de experiencia de sí de carácter reflexivo, la cual se corresponde con el extremo "pensamiento" de la antinomia vitalista. "El 'sentido' de una vivencia señala Schütz- se reduce a un volverse (Zuwendung) hacia una vivencia transcurrida a través del cual la misma es extraída del transcurso de la duración y se torna una vivencia 'tal' constituida-de-tal-o-cual-manera-y-node-otra." En la reflexión, entonces, una vivencia es "captada (erfaßt), diferenciada (unterschieden), destacada (herausgehoben), circunscripta (abgegrenzt)..."38, es decir, el yo "aísla" un estado psíquico del flujo de conciencia tornándolo de este modo discreto y significativo. ${ }^{39}$ Nuevamente puede vislumbrarse con claridad que el concepto de reflexión del que se vale Schütz es idéntico al desarrollado por Husserl. En ambos casos se trata de un acto intencional que se dirige con atención a la propia corriente de conciencia y capta vivencias ya transcurridas; en ambos casos, la retención como "ser-aún-consciente de lo que ha sido inmediatamente-antes (Noch-

36 Schütz, Alfred, Der sinnhafte Aufbau der sozialen Welt, Suhrkamp, Frankfurt am Main, 1981, p. 68.

${ }^{37}$ Ibid., p. 62.

38 Ibid.

39 Ibid., p. 307. 
Bewusstsein des Soebengewesenen)" 40 permite que el acto reflexivo tenga un objeto que captar y no sea un mero discurrir en el vacío.

Ahora bien, los análisis recién expuestos representan sólo un abordaje preeliminar del proceso de autointerpretación. Los mismos describen cómo se constituye el sentido del propio comportamiento en general, esto es, brindan un concepto primero y originario de sentido (erster und ursprünglicher Sinnbegriff) -no hacen más que señalar que éste se constituye en y por la reflexión. Sin embargo, lo que le interesa a Schütz no es dar cuenta del sentido en general sino del sentido especifico (spezifischer Sinn) que cada sujeto le $\mathrm{da}$ a su conducta en un momento y lugar determinados. En otras palabras: Schütz se afana por explicar de qué manera, y por qué, un actor le confiere a su comportamiento este sentido preciso y particular, y no otro. Para ello, el autor austríaco considera que es necesario llevar a cabo una ampliación del primer concepto de sentido, ampliación que realiza sustentándose nuevamente en Husserl. Siguiendo los desarrollos genéticos de la fenomenología husserliana tardía, el sociólogo vienés señala que en el transcurso de su vida cada sujeto sedimenta sus experiencias adquiriendo de este modo un repositorio o stock de experiencia (Erfabrungsvorrat), el cual se convierte en un haber pasivo de la subjetividad y actúa luego como esquema de interpretación (Deutungsschema) de nuevas vivencias. La autointerpretación, como todo proceso interpretativo, implica para Schütz la subsunción (Einordnung) del objeto a interpretar -en este caso, una vivencia propia- bajo uno de los esquemas de experiencia (Schemata der Erfabrung) disponibles, es decir, conlleva un remontar lo nuevo y desconocido a lo ya conocido y sedimentado. Este proceso entonces no se realiza ex nibilo sino que está determinado de modo fundamental por el acervo disponible de experiencias del actor que en cada caso lo lleva a cabo.

Asimismo, Schütz señala que el aquí y así (Jetzt und So) desde el cual se lleva a cabo el acto de autointerpretación condiciona y determina el resultado del mismo. Esta aseveración schütziana se apoya en reflexiones en torno a un elemento teórico fundamental de la fenomenología husserliana: la correlación noético-noemática. Todo cambio en el lado noético conlleva una modificación en el modo de donación (Gegebenheitsweise) del lado noemático. Aquí entran en juego para Schütz las modificaciones en la

40 Ibid., p. 66. 
perspectiva temporal desde la cual se realiza el acto autointerpretativo. Conforme "envejezco" las vivencias retenidas se hunden en la opacidad en virtud de la modificación retencional. Por tanto los estados psíquicos que viví ayer se me donarán de modo diferente dependiendo del punto temporal desde el cual lleve a cabo el acto reflexivo que los recupera. Por otro lado, el ya mencionado stock o repositorio de experiencias se amplía, se modifica y se corrige en cada momento del transcurso de la vida consciente, de modo que los esquemas de interpretación a los cuales se subsumen las vivencias son diferentes dependiendo del momento desde el cual se lleve a cabo el proceso autointerpretativo. $\mathrm{Y}$ algo similar sucede en lo concerniente a las modificaciones atencionales (attentionale Modifikationen). El acto reflexivo puede realizarse con diferentes grados de atención que van desde el captar actualmente (aktuelles Erfassen) hasta el pasar desapercibido (unbeachtet Bleiben), es decir, desde el "prestar suma atención al objeto" hasta el "no prestarle atención alguna". En suma, dado que la autointerpretación no es otra cosa que la captación de una vivencia pasada desde una nueva vivencia, esto es, desde un nuevo ahora y así, el sentido específico del comportamiento —en términos de Weber, el sentido mentado o subjetivo- depende del cómo del volverse (Wie der Zuwendung), es decir, está determinado por todas las modificaciones noéticas recién señaladas.

Estos análisis schützianos de la constitución del sentido subjetivo del propio comportamiento nos habilitan a sacar conclusiones acerca de la postura de Schütz en lo que refiere al primer presupuesto de las teorías dominantes de la empatía criticado por Scheler en Wesen und Formen der Sympathie (I). Schütz parte de la premisa husserliana de que todas las descripciones que se realizan en primera persona sobre las estructuras esenciales de la propia conciencia son igualmente válidas para la conciencia del alter-ego. Esto no implica afirmar que la conciencia del ego y la del alterego sean una y la misma, esto es, que sean empíricamente iguales, sino más bien que cumplen con las mismas leyes esenciales a pesar de que su contenido fáctico difiera. Tal como ego, alter-ego "vive" sus vivencias percatándose pre-reflexivamente de ellas; en base a esta percatación reflexiona sobre las mismas y les confiere un sentido; sus actos autointerpretativos parten de un ahora y así determinado espacial, temporal y atencionalmente; y por último, sedimenta en el transcurso de su vida sus experiencias adquiriendo esquemas interpretativos que utiliza para comprender vivencias nuevas. Todo esto implica que la captación del 
sentido subjetivo del comportamiento ajeno es una empresa imposible: yo no "vivo" ni retengo las vivencias ajenas y tampoco podré contar jamás con exactamente los mismos esquemas interpretativos que él posee ni reproducir exactamente el jetzt und so desde el que realiza su acto autointerpretativo. En resumen: para Schütz, el acceso a subjetividad ajena es extremadamente complejo $y$, aun en condiciones óptimas de interpretación, el sentido subjetivo del comportamiento del alter-ego se mantiene como concepto límite (Limesbegriff). Las diferencias de esta concepción con la de Scheler están a la vista.

(II) Podría afirmarse que Schütz coincide en cierta medida con el segundo presupuesto de las teorías dominantes de la empatía criticado por Scheler en Wesen und Formen der Sympathie - "lo que nos es dado 'ante todo' de otro ser humano es exclusivamente el fenómeno de su cuerpo, sus alteraciones, movimientos, etc." 41 Como se afirmó más arriba, de acuerdo a Scheler no existen diferencias sustanciales entre la autoexperiencia y la experiencia del prójimo: tanto cuando nos percibimos a nosotros mismos como cuando percibimos al alter-ego nos enfrentamos con unidades expresivas, esto es, con totalidades indivisas en las que se amalgaman movimientos corporales y vivencias. Consecuentemente, no es cierto que lo único que podamos conocer del alter-ego sean sus movimientos corporales ni que tengamos acceso inmediato a nuestras vivencias por vía de una experiencia interna pura. Oponiéndose a la postura scheleriana, Schütz se ocupa de remarcar las divergencias existentes entre empatía y autoexperiencia. Siguiendo una vez más a Husserl, el sociólogo vienés considera que la experiencia de sí es un proceso intra-psíquico que no conlleva mediación alguna de la corporalidad y que se lleva a cabo en los dos modos ya explicitados: autopercatación pre-reflexiva y autoconciencia reflexiva. En contraste, es imposible acceder a la conciencia del alter-ego en ninguna de esas dos modalidades. La vida de conciencia del prójimo sólo puede captarse en una experiencia signitiva (signitive Erfabrung) a través del medium de su cuerpo. Éste actúa como campo de expresión (Ausdrucksfeld) de las vivencias ajenas, siendo los movimientos corporales del alter-ego índices (Anzeichen) o síntomas (Symptome) de sus estados psíquicos. ${ }^{42}$

${ }^{41}$ Scheler, Max, op. cit., p. 238.

${ }^{42}$ Cfr. Schütz, Alfred, op. cit. 
En las Logische Untersuchungen, Husserl diferencia tres modos de intencionar un objeto: el signitivo, el pictórico y el perceptivo: "puedo hablar sobre lo horrible que debe ser para un homeless tener que pernoctar en la calle [modo signitivo]; puedo ver un reportaje televisivo sobre eso [modo pictórico]; o puedo probarlo yo mismo [modo perceptivo]." 43 Estas tres modalidades de intencionalidad están ordenadas jerárquicamente de acuerdo a qué tan originariamente nos brindan el objeto intencionado. Es decir, los tres modos noéticos implican, del lado noemático, tres modos de donación del objeto (Weise des Gegebenseins) que van desde la forma más óptima en que éste puede manifestarse, la donación en carne y bueso (leibhaftig) característica de la percepción, a la forma más pobre, la intención vacía propia de los actos signitivos. La percepción es entonces para Husserl la forma madre de la intencionalidad, y los actos pictóricos y signitivos no son más que derivados que remiten siempre en última instancia a ella. Ahora bien, vale preguntarse ¿qué lugar ocupa la empatía dentro de esta clasificación de actos? ¿En qué sitio la ubica Schütz en Der sinnhafte Aufbau der sozialen Welt? Podría afirmarse que para Schütz la empatía se caracteriza por su hibridez: en ella se conjugan elementos propios de la donación intuitiva con otros de la donación signitiva. Siguiendo a Husserl, Schütz señala que en cierto modo es correcto decir que "percibimos" las vivencias de los otros cuando vemos sus movimientos corporales; no obstante, la experiencia de los estados psíquicos ajenos es al mismo tiempo signitiva porque en ella las vivencias del otro no se me donan en carne y bueso sino a través del medium de su cuerpo. En efecto, sólo el alter-ego tiene potencialmente acceso perceptivo a sus propias vivencias a partir de la reflexión; el ego, en cambio, accede únicamente a ellas a través de los síntomas e índices que le brinda la corporalidad ajena. En términos de Husserl: "Experiencia originaria la tenemos de nosotros mismos y de nuestros estados de conciencia en la llamada percepción interna o autopercepción, pero no de los demás ni de sus vivencias en la 'empatía' (Einfüblung). Les "vemos" a los demás sus vivencias [esto es, percibimos sus vivencias] sobre la base de la percepción de sus manifestaciones corporales (leibliche Äußerungen)." 44 ¿Cómo es posible entonces que la empatía sea signitiva y perceptiva a la vez? Es cierto que no

43 Zahavi, Dan, Husserls Phänomenologie, Mohr Siebeck, Tübingen, 2009, p. 29. 44 Husserl, Edmund, Ideen zu einer reinen Phänomenologie und phänomenologischen Philosophie, Felix Meiner, Hamburg, 2009, p. 11. 
podemos percibir las vivencias del alter-ego tal como percibimos las propias, esto es, a partir de una percepción interna o inmanente que nos las brinda en persona, pero esto no implica que no podamos percibirlas en absoluto. En otras palabras: quizás sea posible sostener que estamos capacitados a "percibir" las vivencias ajenas aunque de un modo diferente a como intuimos las propias. Podríamos afirmar, siguiendo a Zahavi, ${ }^{45}$ que no es correcto utilizar el acceso en primera persona que tenemos a nuestros propios estados de conciencia como patrón para medir si percibimos o no las vivencias ajenas. Si hacemos esto condenamos de antemano la viabilidad de un acceso directo, o al menos en cierta medida directo, a los estados psíquicos del alter-ego, ya que es claro que no podemos "vivir" las vivencias del otro ni reflexionar sobre ellas como éste lo hace. Por tanto es preciso reconocer que, así como hay una percepción en primera persona de los propios estados psíquicos, existe también una percepción en segunda persona de las vivencias del prójimo, un acto de conciencia con características particulares que nos brinda los estados psíquicos ajenos de forma directa pero a la vez signitiva.

Schütz considera entonces que la empatía es una experiencia signitivo-simbólica en la cual las vivencias del alter-ego se nos dan a través de su corporalidad. Pero no cree que la "interpretación" de los signos brindados por el cuerpo ajeno implique una actividad del yo ulterior o exterior a la percepción de los mismos, ya sea en forma de un razonamiento por analogía o de otro tipo de operación intelectual. Se trata más bien de una interpretación que se da de forma pasiva, en, y simultáneamente con, la observación del cuerpo ajeno. En el momento de la escritura de Der sinnhafte Aufbau der sozialen Welt, Schütz no había estudiado en profundidad la quinta de las Cartesianische Meditationen, lugar donde Husserl se ocupa del problema de la empatía. No obstante, pueden encontrarse referencias aisladas a ese texto en las notas al pie. Es de destacar la nota 11 que Schütz introduce en el tercer capítulo de su libro. ${ }^{46}$ Allí el sociólogo vienés remite a un fragmento de la quinta meditación donde Husserl da cuenta del modo en que el cuerpo ajeno indica apresentando las vivencias del alter-ego. Esto demuestra que, aun habiendo realizado una lectura superficial de ese texto, el joven Schütz

45 Cfr. Zahavi, Dan, "Empathy, Embodiment and Interpersonal Understanding: From Lipps to Schutz", en Inquiry, vol. 53, N 3, 2010.

46 Ibid., p. 194. 
vislumbra en él algunas coincidencias con su propia concepción del Fremdverstehen. ${ }^{47}$ En la quinta meditación Husserl lleva a cabo desarrollos teóricos que permiten comprender exhaustivamente el carácter dual, signitivo-intuitivo, del proceso de empatía. La noción de Paarung (parificación) como transferencia analogizante o transferencia aperceptiva ocupa aquí un rol central. De acuerdo a Husserl, tal como sucede en cualquier percepción, la empatía implica una apercepción, esto es, un "introducir" - pasivamente, sin necesidad de una actividad del yo- un excedente que va más allá del dato concretamente aprehendido en el ahora actual. Este excedente sería "extraído" -nuevamente, de forma pasiva- de experiencias pasadas sedimentadas que guardan cierta similitud con el objeto presente y "transferido" a éste, lo cual nos permitiría saber más del mismo que lo que actualmente percibimos de él. En una primera instancia, el alterego aparece en mi campo perceptual como cuerpo físico (Körper). Debido a la semejanza que existe entre este Körper y mi cuerpo vivo (Leib), "llevo a cabo" una transferencia aperceptiva en la que "le adjudico" al otro una vida de conciencia estructuralmente análoga a la mía. De esta manera constituyo al cuerpo ajeno como cuerpo de un alter-ego.

Ahora bien, podría señalarse que a pesar de sostener esta concepción bifronte de la empatía, Schütz considera preciso subrayar el carácter signitivo-simbólico de la misma en detrimento de su carácter perceptivo. Esto se debe, según creo, a su enfrentamiento con la teoría scheleriana del Fremdverstehen. En abierto enfrentamiento con Scheler, Schütz se afana por remarcar que de ninguna manera poseemos un acceso inmediato, en carne $y$ bueso, a los estados psíquicos ajenos, es decir, que no podemos experimentar nunca las vivencias del alter-ego tal como él las experimenta. Sólo tenemos acceso a sus movimientos corporales, que actúan como índices (Anzeichen) de sus vivencias, y, si bien la "interpretación" de tales índices se lleve a cabo de modo inmediato en la percepción, a tal punto que podemos decir que "intuimos" o "percibimos" los estados psíquicos del otro, esto no podrá borrar jamás el carácter incierto de la empatía, su incapacidad de captar

47 Es fundamental señalar que en su etapa americana Schütz se desliga de la convicción husserliana de que la Paarung del cuerpo vivo propio con el cuerpo físico ajeno es la base de la empatía impropia (uneigentliche Einfüblung). Cfr. Schütz, Alfred, "Das Problem der Intersubjektivität bei Husserl", en Idem, PhilosophischPbänomenologische Schriften 1. UVK, Konstanz, 2009. 
originaria, certera e infaliblemente las vivencias ajenas. El índice -el movimiento corporal- re-presenta a la cosa -la vivencia del alter-ego- pero jamás nos la brinda en persona. Por tanto, la experiencia que puede tenerse de la vida de conciencia del prójimo es, y será siempre, indirecta, y las afirmaciones que se realicen sobre la misma estarán siempre viciadas por la incertidumbre. Veo a un sujeto realizando determinados gestos e interpreto los mismos como una "sonrisa" y a ésta como índice del agrado que le provoca mi compañía. Pero puedo equivocarme. Es posible que el otro esté fingiendo para hacerme creer que le agrado, y esto debido a fines utilitarios para los que precisa de mi ayuda o complicidad. En otras palabras, es imposible captar las vivencias del prójimo y asir el sentido subjetivo que éste les vincula a partir de la mera observación de sus movimientos corporales.

Ahora bien, para Schütz es justamente esto lo que sostiene Scheler con su concepto de Fremdwabrnehmung. Como señalé más arriba, el convencimiento central de la teoría scheleriana de la empatía es que a una con los movimientos corporales del prójimo se me donan sus estados psíquicos: en su llanto, percibo su tristeza; en su risa, su alegría; en su rubor, su vergüenza, etc. Ante esta posición, vale preguntarse nuevamente con Schütz ¿y si el llanto del otro no expresa su tristeza sino una desbordante felicidad? ¿Qué sucede si simplemente tiene irritada la vista y le "lloran los ojos"? "La mera comprensión actual (aktuelles Verstehen) del proceso exterior — señala Schütz — no puede enseñarme nada acerca de estas cuestiones." 48

De acuerdo a Schütz, el error de Scheler no consiste simplemente en no reparar en el carácter signitivo de la percepción del otro, sino también en confundir fenómenos que deben ser diferenciados claramente. En primer lugar, señala Schütz, es preciso distinguir netamente la interpretación de las propias percepciones, de la comprensión auténtica de las vivencias ajenas (echtes Fremdverstehen). El sociólogo vienés afirma que cuando ego ve a alterego realizando determinado movimiento corporal -lo ve "llorando"- y comprende inmediatamente sus vivencias -"está triste"-, no está

${ }^{48} \mathrm{El}$ concepto de comprensión actual (aktuelles Verstehen) pertenece a Max Weber. Schütz considera que al valerse de este concepto, Weber comete un error análogo al scheleriano. Ambos pensadores creen que es posible captar de forma inmediata los estados psíquicos del prójimo y descuidan por tanto el hiato existente entre la conciencia del ego y la del alter-ego. 
comprendiendo auténticamente al otro sino más bien interpretando las propias percepciones del cuerpo ajeno. Es decir, ego subsume un fenómeno del mundo externo, los movimientos corporales del otro, bajo uno de sus esquemas interpretativos adquiridos colocándole un nombre. Se subsume el objeto percibido a un esquema de interpretación disponible, dependiendo el corolario final de este proceso del Jetzt und So desde el cual se lleva a cabo. Es claro que el resultado de esta interpretación llevada a cabo por ego jamás podrá identificarse con el de la autointerpretación de alter-ego, esto es, con el sentido subjetivo o mentado de la acción. Esto se debe a las razonas explicitadas más arriba: para que mi interpretación de los movimientos corporales de alter-ego se identifique con su autointerpretación, yo debería ser él, esto es, debería haber vivido las mismas experiencias, poseer sus mismos esquemas interpretativos, estar ubicado en el mismo sitio espacial y temporal, etc. "Antes bien, lo que capto en la comprensión actual de los actos (Handlungen) es, por el contrario, la objetividad del proceso de actuar, la cual a partir de un acto de interpretación -algo así como un nombrar (Benennung) - es colocado por mí y para mí en un contexto de sentido (Sinnzusammenhang) que, de ninguna manera, debe ser -más exactamente no puede ser- aquel contexto de sentido que el actor mienta con su actuación". 49 Scheler confunde estos dos fenómenos y parece creer que cuando interpretamos los gestos o ademanes corporales del alter-ego captamos en ese mismo acto el sentido subjetivo de la acción, esto es, comprendemos auténticamente al prójimo. Por eso señala Schütz que la echtes Fremdverstehen implica un cambio radical de actitud, un paso a la actitud-otro (Fremdeinstellung), que se dirige consciente y explícitamente a descifrar los estados psíquicos ajenos, respetando la brecha que existe entre éstos y los propios. Aquí el ego se esfuerza por conocer el verdadero sentido subjetivo del comportamiento del alter-ego, $y$, aunque jamás estará capacitado a asirlo de forma total, sí podrá llegar a valores aproximados (Näherungswerte) al mismo.

\section{Palabras finales}

En este escrito realicé un análisis del debate en torno a la empatía que Alfred Schütz sostiene con Max Scheler en Der sinnbafte Aufbau der sozialen

${ }^{49}$ Ibid., p. 36. 
Welt. Para ello bosquejé en el primer apartado (I. Max Scheler: la teoría de la Fremdwabrnebmung) las críticas que en Wesen und Formen der Sympatbie Scheler le realiza a las dos teorías de la compresión del otro dominantes de su época - la Analogieschlusstheorie y la Theorie der projektiven Einfüblung- prestando especial atención al doble punto de partida común de las mismas. Luego, en el segundo apartado (II. Alfred Schütz: la teoría del Fremdverstehen), intenté mostrar cómo Schütz se enfrenta a la posición scheleriana y elabora su propia teoría del Fremdverstehen concepción sustentado en elementos teóricos husserlianos.

Es preciso dejar asentado que no fue mi propósito aquí realizar una reconstrucción detallada y sistemática de ambas teorías, tarea que excede los límites del presente trabajo, sino más bien contraponer ambas posiciones en sus rasgos más salientes para iluminar las tensiones subyacentes entre las mismas.

Desde mi perspectiva, la revisita y rehabilitación del debate SchützScheler es de importancia decisiva tanto en el campo de la reflexión filosófica como en el de la teoría social, ya que los mojones temáticos que lo estructuran -la relación entre individuo y comunidad, el rol de la corporalidad, las diferencias entre autoexperiencia y conocimiento del prójimo, el atolladero del solipsismo, etc.- son ejes fundamentales a ser tenidos en cuenta por todo abordaje exhaustivo del problema de la empatía.

Recibido: 8/2012. Aceptado: 10/2012 